\title{
ENHO gene expression and serum adropin level in rheumatoid arthritis and systemic lupus erythematosus
}

\author{
Servet Yolbas ${ }^{1, A, D}$, Murat Kara ${ }^{2, C}$, Mehmet Kalaycic ${ }^{3, C}$, Ahmet Yildirimo ${ }^{1, B}$, Baris Gundogdu ${ }^{1, B}$, Suleyman Aydin' ${ }^{3, C}$, Suleyman Serdar Koca ${ }^{1, A, D-F}$ \\ ${ }^{1}$ Department of Rheumatology, Faculty of Medicine, Firat University, Elazig, Turkey \\ ${ }^{2}$ Department of Medical Genetics, Faculty of Medicine, Mugla Sitki Kocman University, Turkey \\ ${ }^{3}$ Department of Biochemistry, Faculty of Medicine, Firat University, Elazig, Turkey \\ A - research concept and design; $\mathrm{B}$ - collection and/or assembly of data; $\mathrm{C}$ - data analysis and interpretation; \\ $D$ - writing the article; $E$ - critical revision of the article; $F$ - final approval of the article
}

\section{Address for correspondence}

Suleyman Serdar Koca

E-mail:kocassk@yahoo.com

Funding sources

None declared

Conflict of interest

None declared

Received on October 28, 2015

Reviewed on December 9, 2015

Accepted on July 18, 2017

\begin{abstract}
Background. Adropin, a secreted protein, is encoded by the energy homeostasis-associated gene (ENHO). It is expressed by a variety of tissues and cells. It has been implicated in several physiological and pathological processes, such as angiogenesis and apoptosis.
\end{abstract}

Objectives. The aim of the present study was to investigate the ENHO gene expression and serum adropin levels in patients with rheumatoid arthritis (RA) and systemic lupus erythematosus (SLE).

Material and methods. The study included 36 patients with RA, 22 patients with SLE and 20 healthy controls (HC). Patients with a disease activity score-28-erythrocyte sedimentation rate (DAS28-ESR) >2.6 in the RA group and an SLEdisease activity index (SLEDAI) $>6$ in the SLE group were accepted as active. Serum adropin levels were analyzed with the enzyme-linked immunosorbent assay (ELISA) method. The ENHO gene and glyceraldehyde 3-phosphate dehydrogenase (GAPDH) gene expressions in peripheral blood mononuclear cells were analyzed with real-time polymerase chain reaction (PCR).

Results. The ENHO gene mRNA expression was significantly higher in the RA group than in the HC group $(p=0.024)$, although it was similar between the SLE and HC groups $(p=0.920)$. On the other hand, there were no significant differences among the study groups in terms of serum adropin levels ( $p>0.05$ for all). Moreover, there was no significant difference in terms of the ENHO expression and serum adropin levels between active and inactive RA and SLE patients.

Conclusions. Although the ENHO gene expression is increased, serum adropin level is not altered in RA. Similarly, adropin seems not to be associated with SLE. However, the potential link between adropin and inflammatory diseases need to be tested in further studies.

Key words: heumatoid arthritis, systemic lupus erythematosus, adropin, energy homeostasis-associated gene

DOI

10.17219/acem/75944

\section{Copyright}

Copyright by Author(s)

This is an article distributed under the terms of the

Creative Commons Attribution Non-Commercial License

(http://creativecommons.org/licenses/by-nc-nd/4.0/) 


\section{Introduction}

Rheumatoid arthritis (RA) is a chronic inflammatory disease characterized by synovitis and damage to the joints. Its prevalence in the general population is around $1 \% .^{1}$ Although the pathological immunological process of the disease is uncertain, many immune cells, such as lymphocytes, macrophages, and leukocytes, and many other molecules, such as cytokines and chemokines, have been shown to play a role in this process. ${ }^{1}$ Angiogenesis also plays a prominent role in pannus tissue formation in RA. ${ }^{1}$ It has been shown that adipocytokines in the peptide structure are associated with many signaling pathways and that pro-inflammatory cytokine release is involved in the pathogenesis of RA. ${ }^{2-4}$ Moreover, they may influence disease phenotype and the course of the disease. ${ }^{2-4}$

Adropin, encoded by the energy homeostasis-associated $(E N H O)$ gene, is a secretory protein playing an active role in energy homeostasis. ${ }^{5}$ The ENHO gene has been determined to be expressed in many organs besides endothelial cells, such as the liver, the brain, the pancreas, and the kidneys. ${ }^{6,7}$ Adropin affects the regulation of glucose and lipid metabolism, energy homeostasis and the modulation of insulin sensitivity. ${ }^{5-7}$

In addition to the metabolic effects, adropin has been shown to have many non-metabolic effect potentials related to angiogenesis, apoptosis and inflammation. ${ }^{5,6,8}$ Adropin has been demonstrated to have an effect on vascular endothelial growth factor receptor (VEGFR), intracellular pathways like PI3K-Akt and ERK1/2, and local and systemic mediators like interleukin 6 (IL-6), which are also effective in RA pathogenesis. ${ }^{5,6,9}$ In addition, while adropin stimulates the critical stages of angiogenesis, such as proliferation, migration and tube formation, it reduces the apoptosis of endothelial cells and vascular permeability. Furthermore, adropin therapy has been shown to ameliorate endothelial function. ${ }^{6}$

These effects of adropin suggest that it may play an active role in the pathophysiology of inflammatory rheumatic diseases. The aim of this study was to evaluate serum adropin levels and the ENHO gene expression in RA and systemic lupus erythematosus (SLE).

\section{Material and methods}

The study included 36 patients with RA, 22 patients with SLE, and 20 healthy controls (HC). The patients fulfilled the established classification criteria. ${ }^{10,11}$ Participants under the age of 18 years or above the age of 80 years, those with signs of infection, and pregnant women were excluded from the study. The protocol of this study was approved by the institutional Ethics Committee, and all the participants gave informed consent before being enrolled in the study. Detailed histories of all the participants were obtained, and systemic and rheumatological examinations were performed. The clinical process and treatments for all participants were also recorded.

Disease activity and/or severity scores were determined by the disease activity score (DAS)-28-erythrocyte sedimentation rate (ESR) in the RA group (patients with a DAS28-ESR score $>2.6$ were considered active); the SLE disease activity index (SLEDAI) and the Systemic Lupus International Collaborating Clinics/American College of Rheumatology damage index (SLICC/ACR) were used in the SLE group (patients with an SLEDAI score $>6$ were considered active). ${ }^{12-14}$

Blood samples were drawn from all the participants, after fasting overnight. Erythrocyte sedimentation rate and $\mathrm{C}$-reactive protein (CRP) levels were assessed by the classic Westergren and immunoturbidimetric methods, respectively. Routine laboratory evaluations of complete blood count, creatinine and total creatinine kinase levels were assessed in all the participants, using standard laboratory methods. Rheumatoid factor (RF) and anti-cyclic citrullinated peptide (anti-CCP) antibody levels were analyzed in the RA group, and RF and anti-CCP titers higher than $15 \mathrm{IU} / \mathrm{mL}$ were considered positive. In addition, autoantibody work-ups (antinuclear antibody - ANA, anti-double stranded DNA (anti-dsDNA) and anti-Sm antibodies) were studied with standard methods in the SLE group on the same day. Antinuclear antibody was detected by the indirect immunofluorescence antibody (IFA) test. Anti-dsDNA and anti-Sm antibodies were measured by the enzyme-linked immunosorbent assay (ELISA), using suitable commercial kits (Euroimmun, Lübeck, Germany).

Serum adropin levels were analyzed by the ELISA method, using an appropriate commercial kit (Cusabio Biotech Co., Wuhan, China).

Total RNA was prepared from peripheral blood cells by the use of a QIAamp RNA Blood Mini kit (Qiagen, Hilden, Germany). Equal amounts of RNA from these samples were reverse transcribed to cDNA, using a Superscript First-Strand cDNA Synthesis Kit (Invitrogen, San Diego, USA). The mRNA expression of ENHO (Qiagen) was quantified and normalized against glyceraldehyde-3-phosphate dehydrogenase (GAPDH). The levels of $E N H O$ and GAPDH were measured by the Rotor-Gene SYBR green-based realtime polymerase chain reaction (PCR), using a real-time PCR system (Rotor-Gene Q; Qiagen). Gene expression was determined by the 2- $\Delta \Delta \mathrm{Ct}$ methodology, normalized against the reference gene GAPDH. Changes in gene expression are represented as a fold change relative to 1 , where the control equals 1 .

Statistical analysis was performed using the Statistical Package for the Social Sciences (SPSS) v. 21.0 (IBM, Chicago, USA). Results were given as mean \pm standard deviation (SD). The normal distribution of the variables was evaluated by the Kolmogorov-Smirnov test, and logarithmic transformations were performed to normalize data with a skewed distribution before statistical analysis. 
Statistical differences among the groups were identified with one-way analysis of variance (ANOVA), followed by Tukey's post hoc test for parametric data and the Mann-Whitney $U$ test for nonparametric data. The $X^{2}$ test was done to compare the categorical variables. Correlation analysis was performed using Pearson's correlation coefficient. Analysis of covariance (ANCOVA) was also used to adjust the variables for age, body mass index (BMI) and current drug usage. The p-values $<0.05$ were considered significant.

\section{Results}

The demographic and clinical data of the study group are summarized in Table 1. The mean DAS28-ESR score was $3.1 \pm 1.6$ in the RA group. The mean SLEDAI and SLICC/ACR indices in the SLE group were $11.3 \pm 10.6$ and $1.7 \pm 1.5$, respectively. There were 17 and 13 active patients in the RA and SLE groups, respectively. In the RA group, the mean swollen, tender and deformed joint counts were $2.5 \pm 4.7,3.8 \pm 6.7$, and $1.1 \pm 2.7$, respectively, and the mean morning stiffness duration was $60.1 \pm 76.2 \mathrm{~min}$. In addition, the mean titers of RF and antiCCP in the RA group were $73.2 \pm 93.3 \mathrm{U} / \mathrm{mL}$ and $363 \pm 50.8 \mathrm{U} / \mathrm{mL}$, respectively, and 19 and 26 RA patients were positive for RF and anti-CCP, respectively. In the SLE group, anti-dsDNA was $78.8 \pm 5.3 \mathrm{IU} / \mathrm{mL}$, and 14 and 6 patients were positive for dsDNA and anti-Sm antibody, respectively.

There was no significant difference among the groups in terms of serum adropin level ( $\mathrm{p}>0.05$ for all). The ENHO gene expression was significantly higher in the RA group when compared to the HC group (1.25 $\pm 0.11 \mathrm{AU}$ vs $1.18 \pm 0.10 \mathrm{AU} ; \mathrm{p}=0.024)$. There was no significant difference between the HC and SLE groups in terms of the ENHO gene expression ( $\mathrm{p}=0.921$ ) (Table 2).

There was no significant difference between active and inactive RA groups in terms of serum adropin and the ENHO gene expression levels ( $\mathrm{p}>0.05$ for all, data not shown). There was no significant difference between RA patients using and not using glucocorticoid (GC), methotrexate, sulfasalazine, hydroxychloroquine (HCQ), and leflunomide in terms of serum adropin levels or the ENHO gene expression. In addition, serum adropin levels and the ENHO gene expression were similar in the patients positive and negative for RF and anti-CCP ( $\mathrm{p}>0.05)$. Furthermore, serum adropin levels and the ENHO gene expression were not significantly correlated with DAS28ESR, ESR, and CRP levels, or RF and anti-CCP titers in the RA group ( $p>0.05)$.

There was no significant difference between the active and inactive SLE subgroups in terms of serum adropin levels and the ENHO gene expression ( $\mathrm{p}>0.05$ for all, data not shown). In the SLE group, there was no significant difference between the patients with and without renal involvement and ANA positivity in terms of serum adropin levels and the ENHO gene expression. Moreover, there was no significant difference between patients using and not using GC, azathioprine and HCQ in terms of serum adropin levels or the ENHO gene expression ( $\mathrm{p}>0.05$ for all, data not shown). However, in the SLE group, the ENHO gene expression was negatively correlated with hemoglobin $(\mathrm{r}=-0.430 ; \mathrm{p}=0.046)$ and low-density lipoprotein (LDL) cholesterol levels $(\mathrm{r}=-0.465 ; \mathrm{p}=0.029)$. 


\section{Discussion}

The current study evaluated serum adropin levels and the ENHO gene expression in RA and SLE, which are chronic inflammatory diseases. There was no significant difference among the groups in terms of serum adropin levels. However, significantly higher $E N H O$ gene expression was observed in RA. On the other hand, the ENHO gene expression was similar between the SLE and HC groups.

Adropin is a molecule in the structure of the peptide and has been shown to act on many chronic pathological processes. Plasma adropin levels increase in patients with heart failure, characterized by chronic, low-grade inflammation. ${ }^{9}$ Furthermore, plasma adropin level shows a positive correlation with IL-6. ${ }^{9}$ On the other hand, Lovren et al. reported that in vitro adropin administration increased Akt and ERK1/2 phosphorylation. ${ }^{6}$ Akt was also shown to interact with several intracellular pathways like GSK3, p21/p27, EDG-1, and FOXO, which were increased as a result of the inflammatory process. ${ }^{6}$ We found increased ENHO gene expression in RA, but not in SLE in our study. Serum adropin levels were not significantly altered in RA and SLE, although they are chronic inflammatory diseases.

Adropin stimulates critical neovascularization processes, including proliferation, migration and capillary-like tube formation. Lovren et al. reported that in vitro adropin administration increased the level of VEGFR2 protein. ${ }^{6}$ Also, an increase in capillary density was observed in the adropin-administered group in a mouse hind limp ischemia model. ${ }^{6}$ Moreover, a low adropin level was shown to be associated with a decrease in vascular microcirculation. ${ }^{15,16}$ In the present study, high levels of the ENHO gene expression in the RA group may suggest that adropin could be associated with pannus formation in RA. In contrast to RA, similar ENHO gene expression between SLE patients and healthy volunteers may be caused by a lack of pannus formation in SLE.

Cardiovascular morbidity and mortality are higher in RA and SLE. ${ }^{17-19}$ The pathogenesis of increased atherosclerosis cannot be explained by common cardiovascular risk factors, such as age, sex, obesity, smoking, hyperlipidemia, hypertension, and diabetes. Inflammation is one of the important non-conventional reasons of increased cardiovascular risk in these inflammatory diseases. In addition, insulin resistance is one of the most important triggering risk factors in the development and progression of atherosclerotic cardiovascular diseases. Adropin is related to metabolic diseases and atherosclerosis. ${ }^{5}$ Increased adipocytes, dyslipidemia, impaired glucose tolerance, and insulin resistance were demonstrated in adropin knockout mice. ${ }^{20}$ Conversely, systemic treatment or transgenic overexpression of adropin were shown to improve obesity, hepatosteatosis and insulin resistance. ${ }^{6,21,22}$ It were shown that a low serum adropin level is associated with endothelial dysfunction and that this dysfunction improves with adropin treatment. ${ }^{6,21,22}$ Increased adropin levels are expected in RA and SLE due to their inflammatory nature. However, in our study, this level was not higher in RA and SLE. It suggests that the adropin level which is not increased may be one cause of the increased metabolic and atherosclerotic complications of RA and SLE.

Liver ENHO gene expression was documented to be affected by changes in one's energy balance, the content of one's diet and the presence of obesity. ${ }^{5}$ While a shortterm diet with a high fat content increases the ENHO gene expression, chronic exposure, through obesity, for example, decreases its expression. ${ }^{5}$ Serum adropin level is high in the case of chow diets and decreases in hunger and diet-induced obesity. While the adropin level is high when fed with a high-fat and low-carbohydrate diet, the adropin level is lower when fed with a low-fat and high-carbohydrate diet. ${ }^{20}$ Additionally, liver ENHO mRNA expression is regulated by liver $\mathrm{X}$ receptors $\alpha(\mathrm{LXR} \alpha)$ and peroxisome proliferator activated receptor- $\gamma$ (PPAR- $\gamma$ ), which is an insulin sensitizer, playing a role in cholesterol and triglyceride metabolism. ${ }^{5}$ The nuclear receptor families, LXR $\alpha$ and PPAR- $\gamma$, playing a role in energy homeostasis, were shown to be higher in RA fibroblast-like synoviocytes and synovial fluid. Also, an increase in LXR $\alpha$ and PPAR- $\gamma$ was demonstrated to be related with a decrease in the ENHO gene expression and the adropin level.,23-26 Thus, it can be concluded that one cause of the adropin level that is not increased may be the possible suppressive effect of LXR $\alpha$ and PPAR- $\gamma$ on adropin production.

Similarly, leptin may suppress adropin production in RA and SLE. A decreased adropin level was observed to be associated with an increased leptin level. ${ }^{5,27}$ The leptin level significantly increases RA and SLE. ${ }^{28,29}$

There are some limitations of this study. Foremost, the sample size is relatively small. The analysis of the ENHO gene expression by peripheral blood mononuclear cells may be another limitation of the present study. It could be analyzed by liver tissue or any affected tissue. Thirdly, another limitation may be that the mean ages of the study groups are significantly different in our study. However, this is difficult to correct, since RA and SLE affect and start at different ages. The differences for data were also analyzed with ANCOVA to adjust.

In conclusion, the ENHO gene expression is increased in RA but not in SLE. However, the adropin level does not change in RA and SLE, which are chronic inflammatory diseases. Consequently, adropin may not be directly related to these diseases. However, further studies are needed to draw a more precise conclusion.

\section{References}

1. Mclnnes IB, Schett $G$. The pathogenesis of rheumatoid arthritis. NEngl J Med. 2011;365:2205-2219.

2. Scotece $M$, Conde J, Gómez R, et al. Beyond fat mass: Exploring the role of adipokines in rheumatic diseases. Sci World J. 2011;11:1932-1947.

3. Krysiak R, Handzlik-Orlik G, Okopien B. The role of adipokines in connective tissue diseases. Eur J Nutr. 2012;51:513-528. 
4. Conde J, Scotece M, López VJ, et al. Adipokines: Novel players in rheumatic diseases. Discov Med. 2013;15:73-83.

5. Kumar KG, Trevaskis JL, Lam DD, et al. Identification of adropin as a secreted factor linking dietary macronutrient intake with energy homeostasis and lipid metabolism. Cell Metab. 2008;8:468-481.

6. Lovren F, Pan Y, Quan A, et al. Adropin is a novel regulator of endothelial function. Circulation. 2010;122:185-192.

7. Aydin S, Kuloglu T, Aydin S, et al. Expression of adropin in rat brain cerebellum, kidneys, heart, liver, and pancreas in streptozotocininduced diabetes. Mol Cell Biochem. 2013;380:73-81.

8. Aydin S, Kuloglu T, Aydin S. Copeptin, adropin and irisin concentrations in breast milk and plasma of healthy women and those with gestational diabetes mellitus. Peptides. 2013;47:66-70.

9. Lian W, Gu X, Qin Y, Zheng X. Elevated plasma levels of adropin in heart failure patients. Intern Med. 2011;50:1523-1527.

10. Aletaha D, Neogi T, Silman AJ, et al. 2010 rheumatoid arthritis classification criteria: An American College of Rheumatology/European League Against Rheumatism collaborative initiative. Ann Rheum Dis. 2010;69:1580-1588.

11. Petri M, Orbai AM, Alarcón GS, et al. Derivation and validation of the Systemic Lupus International Collaborating Clinics classification criteria for systemic lupus erythematosus. Arthritis Rheum. 2012;64:2677-2686.

12. Prevoo ML, van't Hof MA, Kuper HH, et al. Modified disease activity scores that include twenty-eight-joint counts. Development and validation in a prospective longitudinal study of patients with rheumatoid arthritis. Arthritis Rheum. 1995;38:44-48.

13. Bombardier C, Gladman DD, Urowitz MB, et al. Derivation of the SLEDAI. A disease activity index for lupus patients. The Committee on Prognosis Studies in SLE. Arthritis Rheum. 1992;35:630-640.

14. Gladman D, Ginzler E, Goldsmith C, et al. The development and initial validation of the Systemic Lupus International Collaborating Clinics/American College of Rheumatology damage index for systemic lupus erythematosus. Arthritis Rheum. 1996;39:363-369.

15. Ahn J, Kim J. Mechanisms and consequences of inflammatory sig naling in the myocardium. Curr Hypertens Rep. 2012;14(6):510-516.

16. Celik A, Balin M, Kobat MA, et al. Deficiency of a new protein associated with cardiac syndrome $\mathrm{X}$; called adropin. Cardiovasc Ther. 2013;31:174-178.
17. Mason JC, Libby P. Cardiovascular disease in patients with chronic inflammation: Mechanisms underlying premature cardiovascular events in rheumatologic conditions. Eur Heart J. 2015;36(8):482-489c.

18. Ku IA, Imboden JB, Hsue PY, Ganz P. Rheumatoid arthritis: Model of systemic inflammation driving atherosclerosis. Circ J. 2009;73:977-985.

19. McMahon M, Skaggs B. Pathogenesis and treatment of atherosclerosis in lupus. Rheum Dis Clin North Am. 2014;40(3):475-495.

20. Ganesh Kumar K, Zhang J, Gao S, et al. Adropin deficiency is associated with increased adiposity and insulin resistance. Obesity (Silver Spring). 2012;20:1394-1402.

21. Gozal D, Kheirandish-Gozal L, Bhattacharjee R, et al. Circulating adropin concentrations in pediatric obstructive sleep apnea: Potential relevance to endothelial function. J Pediatr. 2013;163:1122-1126.

22. Topuz M, Celik A, Aslantas T, et al. Plasma adropin levels predict endothelial dysfunction like flow-mediated dilatation in patients with type 2 diabetes mellitus. J Investig Med. 2013;61:1161-1164.

23. Palma A, Sainaghi PP, Amoruso A, et al. Peroxisome proliferator-activated receptor-gamma expression in monocytes/macrophages from rheumatoid arthritis patients: Relation to disease activity and therapy efficacy - a pilot study. Rheumatology (Oxford). 2012;51:1942-1952.

24. Zhou JJ, Ma JD, Mo YQ, et al. Down-regulating peroxisome proliferator-activated receptor-gamma coactivator-1beta alleviates the proinflammatory effect of rheumatoid arthritis fibroblast-like synoviocytes through inhibiting extracellular signal-regulated kinase, p38 and nuclear factor-kappa B activation. Arthritis Res Ther. 2014;16:472. https://doi.org/10.1186/s13075-014-0472-6

25. Marder W, Khalatbari S, Myles JD, et al. The peroxisome proliferator activated receptor- $\gamma$ pioglitazone improves vascular function and decreases disease activity in patients with rheumatoid arthritis. J Am Heart Assoc. 2013;2:e000441. doi: 10.1161/JAHA.113.000441

26. Laragione T, Gulko PS. Liver $X$ receptor regulates rheumatoid arthritis fibroblast-like synoviocyte invasiveness, matrix metalloproteinase 2 activation, interleukin-6 and CXCL10. Mol Med. 2012;18:1009-1017.

27. Sayin O, Tokgöz Y, Arslan N. Investigation of adropin and leptin levels in pediatric obesity-related nonalcoholic fatty liver disease. J Pediatr Endocrinol Metab. 2014;27:479-484.

28. Tian G, Liang JN, Wang ZY, Zhou D. Emerging role of leptin in rheumatoid arthritis. Clin Exp Immunol. 2014;177(3):557-570.

29. Vadacca M, Zardi EM, Margiotta D, et al. Leptin, adiponectin and vascular stiffness parameters in women with systemic lupus erythematosus. Intern Emerg Med. 2013;8(8):705-712. 